INSTITUT NATIONAL DE RECHERCHE EN INFORMATIQUE ET EN AUTOMATIQUE

\title{
Dynamic Packet Aggregation to Solve Performance Anomaly in 802.11 Wireless Networks
}

\author{
Tahiry RAZAFINDRALAMBO — Isabelle GUÉRIN-LASSOUS — Luigi IANNONE — \\ Serge FDIDA
}

$\mathbf{N}^{\circ}$ ????

July 2006

Thème COM

apport

derecherche 



\title{
Dynamic Packet Aggregation to Solve Performance Anomaly in 802.11 Wireless Networks
}

\author{
Tahiry RAZAFINDRALAMBO*, Isabelle GUÉRIN-LASSOUS ${ }^{\dagger}$, Luigi \\ IANNONE $^{\ddagger}$, Serge FDIDA ${ }^{\S}$ \\ Thème COM — Systèmes communicants \\ Projets ARES
}

Rapport de recherche $\mathrm{n}^{\circ}$ ???? — July 2006 - 28 pages

\begin{abstract}
In the widely used 802.11 standard, the so called performance anomaly is a well known issue. Several works have tried to solve this problem by introducing mechanisms such as packet fragmentation, backoff adaptation, or packet aggregation during a fixed time interval. In this paper, we propose a novel approach solving the performance anomaly problem by packet aggregation using a dynamic time interval, which depends on the busy time of the wireless medium. Our solution differs from other proposition in the literature because of this dynamic time interval, which allows increasing fairness, reactivity, and in some cases efficiency. In this article, we emphasize the performance evaluation of our proposal.
\end{abstract}

Key-words: Wireless Networks; IEEE 802.11; Performance Anomaly.

* tahiry.razafindralambo@insa-lyon.fr

$\dagger$ isabelle.guerin-lassous@insa-lyon.fr

¥ luigi.iannone@lip6.fr

$\S$ serge.fdida@lip6.fr 


\section{Agrégation Dynamique de Paquets pour Résoudre l'Anomalie de Performance des Réseaux sans Fils 802.11}

Résumé : L'anomalie de performance est un problème bien connu du standard 802.11. Il est aussi l'un des plus étudiés. Ces dernières années des solutions permettant de résoudre ce problâA"me, telles que la fragmentation de paquet, l'adaptation de l'algorithme de backoff, ou l'agrégation d'envois de paquets durant un temps donné, ont été developpées. Dans ce papier nous proposons une solution au problème de l'anomalie de performance basée sur une agrégation des paquets en utilisant un intervalle de temps dynamique, qui dépend du temps d'occupation du médium sans fil. Cette approche dynamique nous permet d'augmenter l'équité, la réactivité, et d'être dans certain cas plus efficace comparé aux autres solutions proposées dans la littérature.

Mots-clés : Réseaux sans fil; IEEE 802.11; Anomalie de Performance. 


\section{Introduction}

Performance anomaly is a key issue in IEEE 802.11 multi-rate wireless networks. It decreases the network global performance because of a bad time sharing between stations transmitting at high bit rate (fast stations) and stations transmitting at slow bit rate (slow stations). This bad time sharing results in an unfair throughput, with slow stations throttling fast stations' traffic 4. Several solutions have been proposed in the literature to solve this problem. Some of them are based on a static and predefined time sharing between slow and fast stations by shaping the MTU (Maximum Transmission Unit) on a transmission rate basis. Other approaches set the maximum amount of time a station can hold the medium, like with the TXOP (transmit opportunity) introduced in the IEEE 802.11e standard. Finally, other approaches try to adapt the contention window size of IEEE 802.11, accordingly to the transmission rate of the station.

The main problem of existing solutions is that they are static or centralized. In this paper, we tackle both issues, solving the performance anomaly with a dynamic and distributed approach. Our solution is dynamic because it introduces a transmission time, similar to the TXOP, that changes depending on the perceived channel occupancy, which in turns evolves with the traffic load of the network. Our solution is a distributed approach because each node computes locally the maximal channel occupancy time, based on the active medium sensing provided by IEEE 802.11. Once a node gains access to the medium, it can send as many packets as allowed by the computed transmission time depending on the sensed maximal channel occupancy time.

In this article, we emphasize the performance evaluation of our approach. We propose an analytical evaluation of our protocol in the classical scenario where all stations are within communication range and a detailed simulation-based evaluation. We evaluate our protocol in terms of efficiency and of fairness on many configurations not limited to one-hop networks. We also compare our solution to three different approaches that belong to the three main classes of solutions solving the performance anomaly.

The remaining of the paper is organized as follow. We give a short overview on the IEEE 802.11 access function and describe the performance anomaly in Section 2 In Section 3 we propose a review of the existing modifications of the IEEE 802.11 that solve the performance anomaly. In Section 4 we describe our proposal. In Section 5 we propose an analytical evaluation for a specific topology while in Section 6 we describes the simulations carried out to evaluate the performances and the impact of the different parameters of the proposed protocol on various scenarios. Finally, we conclude the paper with the perspectives raised by this work in Section 7

\section{The Performance Anomaly}

The IEEE 802.11 standard $[3$ provides a totally distributed medium access protocol, called the Distributed Coordination Function (DCF). The DCF is part of the Carrier Sense Multiple Access with Collision Avoidance (CSMA/CA) family. Emitters have to wait for the channel 
to become free before sending a frame. When a frame is ready to be emitted, it is emitted after a fixed time interval called the DIFS (Distributed Inter Frame Space) during which the medium shall stay idle. If the medium is or becomes busy during this interval, a random number called backoff out of an interval called Contention Window $(C W)$ is generated. This number indicates the time to be waited before transmitting. When the medium becomes idle again, the mobile waits for a DIFS before starting to decrement its backoff. When the medium becomes busy during the decrease, the process is stopped and will be resumed later after a new DIFS with the remaining backoff. As soon as the backoff reaches 0 , the frame is emitted. Since collision detection is not possible, each unicast frame has to be acknowledged. When a receiver successfully receives a frame, it waits for a SIFS (Short Inter Frame Space) time and then emits the acknowledgment. The SIFS is shorter than the DIFS in order to give priority to acknowledgments over data frames. The lack of the reception of an acknowledgment is considered as a collision. In that case, the $C W$ size is doubled and the same frame is re-emitted with the same process described previously. If another collision happens, the $C W$ size is doubled again if it has not yet reached the maximum value defined by the standard. After a fixed number of retransmissions, the frame is dropped and the $C W$ size is reset, as for a successful transmission.

Heusse et Al. 4] have shown that the presence of slow terminals in a multi-rate wireless network slows down every other terminal. During the transmission of a slow terminal the medium is busy for a longer period than during the transmission of a fast terminal. Since 802.11 provides simple per-packet fairness in one-hop networks, meaning that in a long period, each emitter statistically has sent the same number of frames. On a time basis, however, slow terminals have occupied the channel for a longer period of time. This time unfairness that arise as soon as multiple rates are present, can lead to a loss of performance due to the existence of slow transmissions.

\section{Related work}

By letting both fast and slow stations to capture the channel for the same amount of time, the performance of IEEE 802.11 should be improved. The issue has been tackled in several different ways, with solutions placed at different levels of the protocols stack. Here we present the most relevant works that try to solve the performance anomaly by introducing tiny modifications in the IEEE 802.11 standard itself, as we do in our solution.

In this context, there exist three main approaches: packet fragmentation, contention window adaptation and packet aggregation. In the following subsections, we describe briefly each approach and we give few relevant examples to illustrate this state of the art.

\section{Packet Fragmentation Approach}

Packet fragmentation is the first and simplest approach. Iannone et Al. 6 propose a solution based on a virtual time division scheme that reduces the performance anomaly of IEEE 802.11. In this solution packets of higher layers are fragmented according to the transmission rate at which they are sent at the 802.11 MAC level. The packet fragment size is fixed and computed offline. Simulation results, presented in that work, show that this 
solution reduces performance anomaly while increasing global throughput. Nevertheless, the static nature of the proposed solution is efficient only for stations transmitting at the higher bit rate with a packet size equal to the MTU on the network. The performance of the network decreases when only slow hosts are present in the network, due to the overhead introduced by the high level of fragmentation in small packets. A similar approach is proposed by Dunn et Al. [2], but at a higher level. The MTU discovery process is used to determine the packet size according to the data rate. This solution has the same poor performance of the previous when only slow hosts are present in the network.

\section{Contention Window Adaptation Approach}

The second category of solution is based on the modification of the backoff mechanism, in particular changing the contention window $(C W)$ size. Heusse et Al. [5] propose a two-step mechanism scheme based on the station data rate. The first step is a protocol that tries to reach an optimal $C W$ size. This optimal value $\left(C W_{\text {opt }}\right)$ is computed according to the number of idle slots perceived on the medium by the station. Then, in a second step, this $C W_{\text {opt }}$ is modified according to the data rate of the station and the maximum available data rate of the network. The proposed protocol reduces the performance anomaly while improving the throughput. The authors show that the main issue of the protocol is the way to compute the optimal windows. The optimal windows values are computed offline according to a fixed data rate. Another problem that can be encountered with this protocol is the long convergence time especially when stations are mobile.

\section{Packet Aggregation Approach}

The third and last category is the packet aggregation approach, in which our solution is also included. This type of solution was first introduced by Sadeghi et Al. [10]. The authors propose an opportunistic media access for multi-rate ad hoc networks. The solution is based on the fact that a station transmitting at high data rate likely to have good channel condition and thus is allowed to send more than one packet to take advantage of this favorable channel condition. The number of successive packets to transmit is computed according to the basic rate of the network. For example if the basic rate is $2 \mathrm{Mbps}$ and the channel condition is sensed such that transmission at $11 \mathrm{Mbps}$ is feasible, the sender is granted a channel access time sufficient to send $11 \% 2=5$ packets. With this solution, performance anomaly can be solved. However, if there are only fast stations on the network, short term unfairness appears.

The packet aggregation solution is also proposed in the IEEE 802.11 e standard [8]. In IEEE 802.11e, a transmission opportunity (TXOP), i.e. a maximum channel occupation time, is granted to every station. This transmission opportunity is broadcasted by the base station to every node. The computation of TXOP is not really clear in the standard, and, as far as we know, it is computed according to the time needed to send the MTU at the lowest data rate. Thus during a TXOP fast stations can aggregate their packets, while slow stations can only send one packet. The main problem of IEEE $802.11 \mathrm{e}$ is that it is centralized. Another problem with a static packet aggregation is that the performance anomaly is solved on one hand but short time unfairness may arise on the other hand.

RR $n^{\circ} 0123456789$ 
To solve the performance anomaly and at the same time this possible short time unfairness issue, we propose a dynamic packet aggregation policy. Our solution is different from the other aggregation solutions because it is not centralized but totally distributed and because it is not static but totally dynamic. The transmission time is computed dynamically at each node, according to simple information perceived on the medium as we will describe it on the next section. Our approach does not need any additional information except those provided by IEEE 802.11 .

\section{PAS: a dynamic packet aggregation}

The idea of our protocol, called PAS (Performance Anomaly Solution), is based on the fact that each station should have the same transmission time on the radio channel. Therefore, if an emitter senses a channel occupancy time that is longer than the transmission time of the packet to be emitted, then it can aggregate packets in order to get a better channel occupancy time. The aggregation is realized by spacing the reception of the previous packet's acknowledgment and the emission of the next packet with a SIFS. There are two main mechanisms in PAS: the first one is the medium sensing that computes the transmission time; the second one is the packets sending, based on the transmission time computed previously.

\subsection{Computing the transmission time}

The first mechanism for the computation of the allowed transmission time is given in Algorithm 1 A station always senses the radio medium and maintains the channel occupancy time. This time is the time during which the channel is sensed busy due to a transmission, including transmission that can be only sensed but not decoded (i.e. in the carrier sensing area). The maximum channel occupancy time is maintained by each station in a variable called $t \_p \_$max. This parameter is set to 0 after each successful transmission of the station. This avoids the station to monopolize the channel after a transmission and improves the reactivity of the protocol. Furthermore, this mechanism allows to reduce the short time unfairness that can be introduced when the same node successively accesses the radio channel.

Note that with this approach, the computed transmission time will never correspond to the time required for an exchange of packets like Data-ACK or RTS-CTS-Data-ACK, since this time is deduced from a continuous signal and will be recomputed as soon as there is a silence period. Moreover, it is very difficult to determine these exchanges times since our computation takes into account signals in the carrier sensing area and that it is not always possible to distinguish a control packet (RTS, CTS or ACK) from a data packet with the same transmission time. 


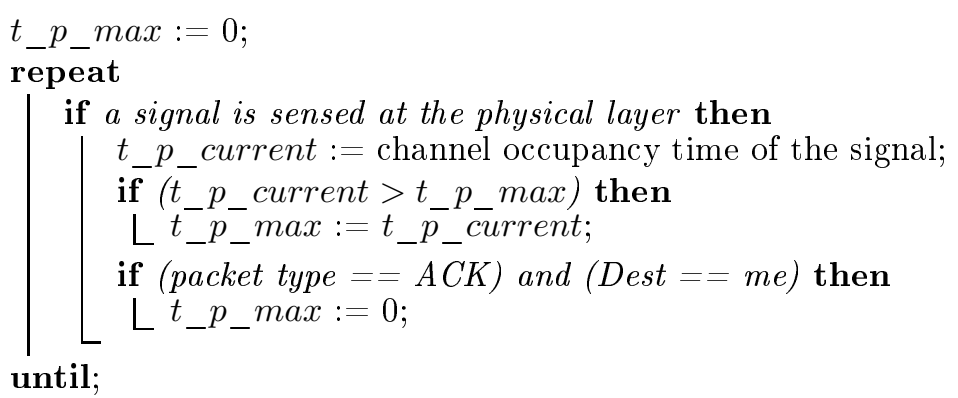

Algorithm 1: Performance Anomaly Solution - Sensing Phase

\subsection{Packet emission}

The second mechanism concerns the emission phase and is given in Algorithm 2. The station can either transmit its packet classically by using the medium access mode of IEEE 802.11 or aggregate some of its packets. To know whether it can aggregate or not, it uses the parameter $t \_p \_m a x:$ if its channel occupancy time is smaller than the value of this variable, then it can aggregate. In Algorithm 2 $t \_m y \_p a c k e t$ is the time required to send the current packet, while $t$ tmy_left corresponds to the remaining allowed transmission time. The value of this last parameter evolves with time and with the packets previously emitted. When this value becomes too small, no more aggregation is possible, otherwise the medium occupancy time of this station would become larger than the maximum transmission time sensed on the channel, which is not fair.

The boolean variable sending indicates whether the packet to send is the first packet to be emitted or not. If it is the first (sending set to false), the packet has to be emitted with the classical medium access of IEEE 802.11. If it belongs to an aggregated packets series (sending set to true), in this case two consecutive packets are only separated with a SIFS.

The parameter $\alpha$ is used to maintain a good overall throughput. Indeed, let consider a scenario with two emitters, one at $11 \mathrm{Mbps}$ and one at $5.5 \mathrm{Mbps}$. These two emitters send packets of the same size. Due to the physical header overhead (the physical header is sent at the same rate whatever the emission rate), the time for transmitting two packets at $11 \mathrm{Mbps}$ is a little bit longer than the time for transmitting one packet at 5.5Mbps. Therefore, without the use of the variable $\alpha$, the fast station will never aggregate and the performance anomaly will remain present. By choosing:

$$
\alpha=\left(\left\lceil\frac{t \_m y \_l e f t}{t t_{-} m y \_p a c k e t}\right\rceil-\frac{t \_m y \_l e f t}{t \_m y \_p a c k e t}\right) * t \_m y \_p a c k e t
$$

packet aggregation and good aggregated throughput is ensured, due to the over-approximation of the transmission time. Note that this parameter is the smallest over-approximation of the transmission time. A new value of $\alpha$ is computed at each new packet arrival at the MAC layer. Thus, we have a real dynamic approach adapted to the current traffic. Furthermore, such an approach does not require a specific assumption on the packet size. 
If a collision occurs on a packet sent with the classical medium access of IEEE 802.11, then the collision resolution mechanism of IEEE 802.11 is applied. If a collision occurs on a packet sent on an aggregated packets series, then the transmission is deferred after a SIFS if $t$ my_left is large enough to send the packet again. Otherwise if $t \_m y \_l e f t$ is too small, the backoff window size is increased according to the binary exponential backoff scheme and sending is set to false, while $t_{-} m y \_l e f t$ is set to 0 . In the sake of simplicity and due to space constrains, the collision part is omitted.

\subsection{Further Improvement}

The transmission time is determined by computing on line the number of packets that can be emitted and whose total time corresponds to the maximum channel occupancy perceived on the channel. The transmission time of one packet includes the time to transmit the packet header. Therefore, if a fast station aggregates many small packets, then a lot of time is lost due to overhead and the overall throughput of network may not be very good. To improve the overall throughput, it is possible to penalize the stations that send small packets. An easy way to do it is to compute the ratio between packet payload and packet

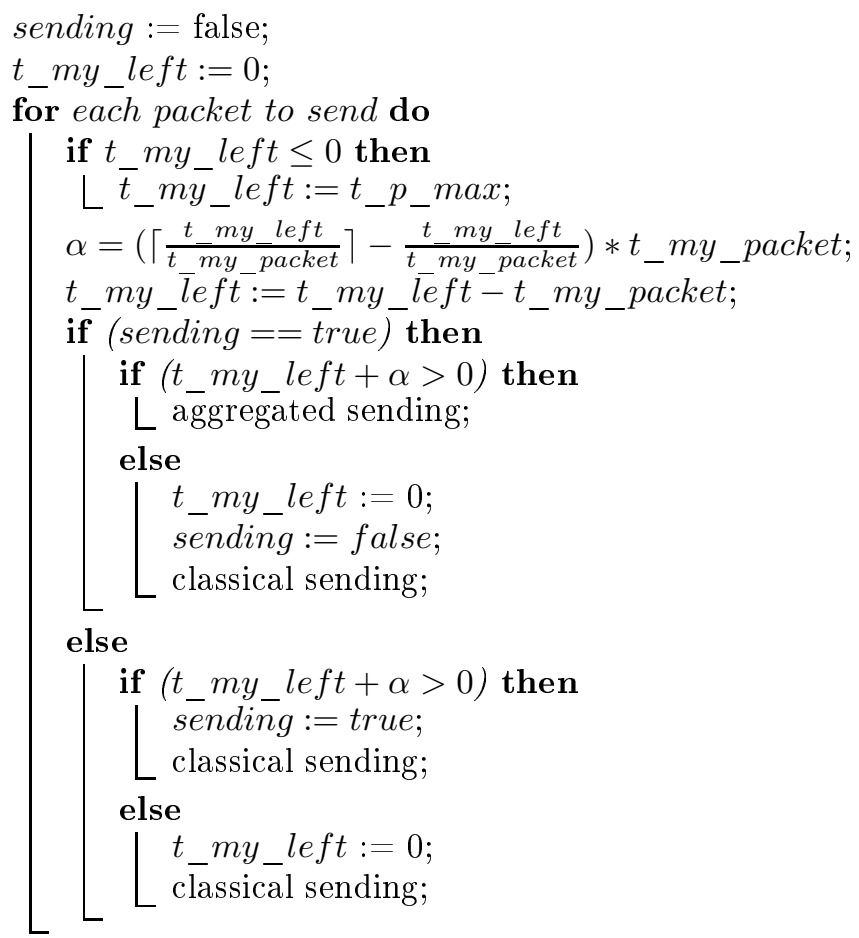

Algorithm 2: Performance Anomaly Solution - Emission Phase 
header (including acknowledgement), we call this ratio $t_{-}$rate, and to use this parameter to limit the aggregation. In our proposition (PAS), the computation of the next value of $t_{-} m y \_l e f t$ is conditioned by the value of $t$ rate. For instance, if $t$ _rate $<1, t_{-} m y \_l e f t=$ $t_{-} m y \_l e f t-\left(\left(1 / t \_\right.\right.$rate $\left.) * t \_m y \_p a c k e t\right)$. At each step this test will reduce the time left for the aggregation of a station that sends small packets. If at the next step, the packet does not satisfy this test, $t_{-} m y \_l e f t$ is then computed normally.

In order for to be compatible with all the 802.11 features, it must work also in presence of RTS/CTS. In this case, PAS uses the duration time given in RTS and CTS frames to update its maximum occupancy time if this duration time is greater than the maximum occupancy time computed previsously. The parameter $t_{-} m y \_l e f t$ is still computed like in Algorithm 2 . Considering transmission, when $t \_p \_m a x \geq t \_m y \_p a c k e t$ and packet $_{\text {length }} \geq R T S_{\text {thresh }}$, then the exchange is as follow: RTS-CTS-DATA-ACK-SIFS-DATA-ACK.... The duration time in the RTS and CTS is the duration for only one packet transmission. There are two reasons to not put the value of $t_{-} p_{-} \max$ in the duration field of the RTS and CTS frames: i) since the number of packets in the LL queue is not known a priori when a RTS is sent, it is possible that the emitter will not use its whole transmission time, which will unnecessarily stop some potential emitters; ii) reactivity is improved. If we assume two fast stations and one slow station, the two fast stations may aggregate their packets based on the transmission time of the slow station. If the slow station stops emitting, the two fast stations will maintain their aggregation because the duration field remains the same for these two stations.

With PAS, collisions, when RTS/CTS mechanism is used, are solved in the following way. If a collision occurs on a RTS, the RTS is retransmitted according to IEEE 802.11, i.e. after a backoff window incrementation. When a collision occurs on the data, the data packet is sent after a SIFS, if $t_{-} m y \_l e f t$ is large enough to send the packet again. If $t_{-} m y \_l e f t$ is not large enough, then a RTS is sent after a backoff window incrementation.

\section{A theoretical analysis}

In this section, we investigate the efficiency and the fairness achieved by PAS. Tan et Al. 11 have proposed the notion of time-based fairness that gives to each node an approximately equal occupancy of the channel. They show that a mechanism that provides a time-based fairness is more efficient than a mechanism that is fair in the medium access. The solution they propose ${ }^{1}$ takes into account the time required for the exchange data-ACK in the computation of the transmission time, whereas PAS is based on the maximum channel occupancy that can never be such an exchange. In this section, we show that PAS is more efficient than solutions based on data-ACK exchanges and we study the time-based fairness of PAS.

\footnotetext{
${ }^{1}$ The work has not been described in Section 3 since the solution is also considered at upper layers and not only at the MAC layer.
} 


\section{$5.1 \quad$ Efficiency}

The time transmission in our protocol is based on packet time and not on the time required for an exchange. An exchange time can be defined as $T_{-} e x=t \_m y \_p a c k e t+T \_S I F S+$ $T \_P H Y+T \_A C K$, where $T \_S F I S$ is the duration of a SIF $\bar{S}, T_{-} P H Y$ is the duration of the PHY header and $T A C \bar{K}$ is the time duration of an ACK. By $t p$ max we denote the maximum channel occupancy time, by $t \_m y \_p a c k e t$ the time required to transmit the packet, and by $T$ ack the sum of $T \_S I \bar{F} S+\bar{T} P H Y+T A A C K$. We assume that $T \_a c k$ is independent from the data rate at which a node transmits and is a constant. We also assume as scenario two stations within communication range from each other (one fast station and one slow station) that use the same packet length. The maximum aggregate throughput is obtained when the fast station aggregate as much packets as possible, on the basis of the transmission time of the slow station. The number of packets sent by the fast station with PAS is given by:

$$
n_{a}=\frac{t \_p \_ \text {max }}{t \_m y \_p a c k e t}
$$

while the number of packets sent by the fast station using the exchange time for the aggregation, like in the work of Tan et Al. 11, is given by:

$$
n_{e t}=\frac{t \_p \_m a x+T \_a c k}{t \_m y \_p a c k e t+T \_a c k}
$$

We have $t \_m y \_p a c k e t \leq t \_p \_$max. Thus, with these assumptions:

$$
n_{a} \geq n_{e t}
$$

Therefore, each time the slow station sends a packet, the fast station, in its next transmission, will aggregate more packets with PAS than with the solution proposed by Tan et Al. [11, showing the higher efficiency of PAS.

\subsection{Fairness}

In this section, we investigate the time-based fairness as discussed by Tan et Al.. In the sake of simplicity, in this analysis we assume that each node uses the same packet length $L$ (in bytes). We also assume that $T_{i}$ with $i=1,2,5.5,11$ is the time needed to transmit a packet at data rate $i \mathrm{Mbps}$. $T_{i}$ includes the transport layer header, the network layer header, the MAC layer header and PHY layer header. We can easily compute the time used by a station transmitting at a data rate $i$ as:

$$
A g g_{i}=n_{a_{i}} \times\left(T_{i}+T_{-} a c k\right)+\left(n_{a_{i}}-1\right) \times T \_S I F S
$$

$A g g_{i}$ is the time required for the aggregated transmission of a node transmitting at data rate $i$, where $n_{a_{i}}=t_{-} p_{-} \max / T_{i}$. From the medium point of view, the time proportion used for an aggregated transmission of one node is:

$$
O c c_{i}=\frac{A g g_{i}}{\sum_{j}\left(A g g_{j} \times N_{j}\right)+N * D I F S}
$$


where $N_{j}$ is the number of stations transmitting at a data rate $j$, with $\sum_{j} N_{j}=N$. We assume here that the probability to access the medium is the same for all the nodes and that during a time interval, each node has accessed the medium exactly once. The number of packets sent by a node transmitting at a data rate $i$, in a time interval $t$, is:

$$
N B p_{i}=\frac{n_{a_{i}}}{\sum_{j}\left(A g g_{j} \times N_{j}\right)+N \times\left(D I F S+A v g_{b c k f}\right)} \times t
$$

where $A v g_{b c k f}$ is the average backoff. We can thus derive the average throughput in bps of a station transmitting at a data rate $i$ with the following equation:

$$
T H_{i}=N B p_{i} \times L \times 8
$$

All the above results can be applied with different packet sizes, the main parameter to know is $t \_p \_m a x$. In this analysis, we assume that stations access to the medium in a TDMA mode, i.e. one station after the other. This assumption is legitimate due the fair access provided by the backoff scheme implemented in the DCF of IEEE 802.11. However, we will see, in the following section, that there are some small differences between the analytical results and the simulation results and that these differences come from this assumption. Indeed, IEEE 802.11 does not provide a perfect TDMA scheduling in the short-term.

Figure 1 shows, for two stations, the proportion of medium occupancy time. One of the two stations transmits at $11 \mathrm{Mbps}$ while the other transmits at $1,2,5.5$, or $11 \mathrm{Mbps}$ (on the $\mathrm{x}$-axis, $i$ Mbps indicates that one station emits at $i$ Mbps while the other emits at $11 \mathrm{Mbps})$. Packet size is equal to 1000 bytes. For each $i$, this figure gives the proportions of medium occupancy time of the fast station (11Mbps) and of the slow station ( $i \mathrm{Mbps})$ and the time proportion when the medium is free. We can see that the fast station gets a larger proportion of medium occupancy than the slow station and that the proportion of each station is not $50 \%$ as it should be with a perfect time-based fairness. This difference may be easily explained by the fact that the allowed transmission time computed with PAS does not take into account the acknowledgments that consume transmission time. We can also see from this figure that the higher the data rate of the slow station, the higher the proportion of medium free. This is due to the proportion between the backoff time and the medium occupancy time that increases.

Table [1] shows the throughput obtained by Equation 8. We included the Jain fairness index [9] to evaluate the fairness of our solution. The Jain index is defined as $\frac{\left(\sum_{i} r_{i} / r_{i}^{*}\right)^{2}}{n \sum_{i}\left(r_{i} / r_{i}^{*}\right)^{2}}$, where $r_{i}$ is the rate achieved on flow $i, n$ is the number of flows, and $r_{i}^{*}$ is the reference rate on flow $i$. As reference rate we use the one defined by Tan et Al. . This rate $r_{i}^{*}$ is computed as if all the flows in the wireless networks were emitted at the same data rate as flow $i$. For example, if we consider two nodes transmitting at 11 (flow 1) and 1 Mbps (flow 2). Then $r_{1}^{*}$ will be the throughput of flow 1 if flow 2 is transmitted at $11 \mathrm{Mbps}$. In the same way, $r_{2}^{*}$ will be the throughput of flow 2 if flow 1 is transmitted at $1 \mathrm{Mbps}$. The value of $r_{i}^{*}$ is the throughput value when the medium occupancy time is equal for all nodes. This is the reason why the index computed in table 1 are not equal to 1. 


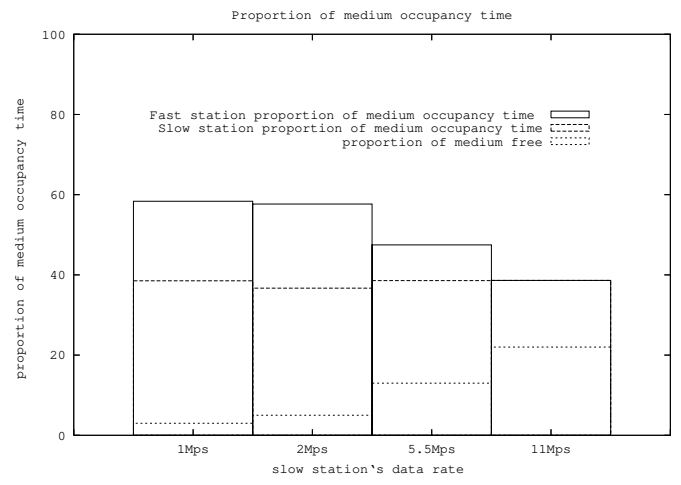

Figure 1: Proportion of medium occupancy time for two stations

\begin{tabular}{|c|c|c|c|}
\hline & Th. (kbps) & Pkt nb. (/s) & Index \\
\hline $5.5 \mathrm{Mbps}$ & 1547.2 & 193.4 & 0.98 \\
11Mbps & 3095.2 & 386.9 & \\
\hline 2Mbps & 624.8 & 78.1 & 0.93 \\
11Mbps & 3749.6 & 468.7 & \\
\hline 1Mbps & 344.8 & 43.1 & 0.92 \\
$11 \mathrm{Mbps}$ & 3791.2 & 473.9 & \\
\hline
\end{tabular}

Table 1: PAS: analytical results 


\begin{tabular}{|l|c|c|c|}
\hline & & Th. $(\mathrm{kbps})$ & Conf. Int. (0.05) \\
\hline \multirow{3}{*}{802.11} & $11 \mathrm{Mbps}$ & 2747.04 & {$[2731.35 ; 2762.72]$} \\
& $11 \mathrm{Mbps}$ & 2752.80 & {$[2736.80 ; 2768.81]$} \\
& Total & 5499.84 & {$[5491.02 ; 5508.66]$} \\
\cline { 3 - 4 } & Index & \multicolumn{2}{|c|}{0.99999} \\
\hline \multirow{3}{*}{ PAS } & $11 \mathrm{Mbps}$ & 2740.61 & {$[2726.91 ; 2754.30]$} \\
& $11 \mathrm{Mbps}$ & 2753.71 & {$[2740.51 ; 2766.92]$} \\
& Total & 5494.32 & {$[5485.78 ; 5502.86]$} \\
\cline { 3 - 4 } & Index & \multicolumn{2}{|c|}{0.99999} \\
\hline \multirow{3}{*}{ Theoretical } & $11 \mathrm{Mbps}$ & \multicolumn{2}{|c|}{$2802.5919(\mathrm{kbps})$} \\
& $11 \mathrm{Mbps}$ & \multicolumn{2}{|c}{$2802.5919(\mathrm{kbps})$} \\
& Total & \multicolumn{2}{|c}{$5605.1839(\mathrm{kbps})$} \\
\hline
\end{tabular}

Table 2: Model validation

\section{Simulations results}

The NS-2 simulator [7] is used to evaluate PAS, which is coded as an independent MAC. Multi-rate features are also added to the simulator, in order to reflect the IEEE 802.11 modulations. All the studies listed below are done in steady state condition. In order to reduce the simulation time and to better evaluate the protocol, ARP and routing protocol exchanges are disabled. In all simulations UDP saturated traffic is used. If not differently specified, each packet contains 1000 bytes of data. Nevertheless, we also developed a module to generate packets of a random size, uniformly distributed in a specific interval.

\subsection{Model validation}

In order to validate the improvements to NS-2 and the code of our proposal, we first simulate two pairs of station transmitting at $11 \mathrm{Mbps}$ with 1000 bytes of data. In this simulation, no aggregation is done because the maximum occupancy time perceived by each node is equal to the time required to send a packet. In this specific case, the throughput of 802.11 and PAS should be the same. This is confirmed by the results presented in Table 2 which includes the theoretical throughput derived in Section $[$ in order to show the accuracy of our model.

\subsection{Basic simulations}

This section contains the first simulation results of PAS. The simulation carried out is based on the classical scenario where two stations transmit packets of 1000 bytes, one at xMbps (x equal to 1,2 or 5.5) and the other at 11Mbps. Tables [3 [ and 5 give the simulation results in this scenario. In these tables, we give the achieved throughput of each station,

RR $n^{\circ} 0123456789$ 
the achieved overall throughput, the number of sent packets by each station and in total, as well as the Jain fairness index, introduced in Section 5

One can see from these tables that the aggregate throughput of PAS is always greater than 802.11, thus PAS is more efficient. It can also be observed that when using PAS, the number of packets and the throughput of the fast station remain almost the same, independently of the rate used by the slow station. This is because the time occupation is roughly divided by 2 between the fast station and the slow station. The fairness index shows that PAS achieves a very good fairness in terms of medium occupancy in these scenarios.

\begin{tabular}{|c|c|c|c|c|c|c|}
\hline & & Th. $(\mathrm{kbps})$ & Conf. Int. $(0.05)$ & Packets/s & Conf. Int. $(0.05)$ & Fairness index \\
\hline \multirow{3}{*}{82.11} & $5.5 \mathrm{Mbps}$ & 2157.02 & {$[2147.86 ; 2166.19]$} & 258.79 & {$[257.34 ; 260.24]$} & \\
& $11 \mathrm{Mbps}$ & 2111.78 & {$[2099.96 ; 2123.61]$} & 264.34 & {$[263.21 ; 265.46]$} & 0.9556825 \\
& Total & 4268.81 & {$[4260.53 ; 4277.10]$} & 523.13 & {$[522.12 ; 524.15]$} & \\
\hline \multirow{3}{*}{ PAS } & $5.5 \mathrm{Mbps}$ & 1769.89 & {$[1761.23 ; 1778.54]$} & 216.89 & {$[215.83 ; 217.95]$} & \multirow{2}{*}{0.9978824} \\
& $11 \mathrm{Mbps}$ & 2943.07 & {$[2927.82 ; 2958.32]$} & 360.67 & {$[358.80 ; 362.53]$} & \\
& Total & 4712.96 & {$[4703.02 ; 4722.91]$} & 577.56 & {$[576.35 ; 578.78]$} & \\
\hline
\end{tabular}

Table 3: Performance anomaly results (throughput and number of packets)

\begin{tabular}{|c|c|c|c|c|c|c|}
\hline & & Th. (kbps) & Conf. Int. $(0.05)$ & Packets/s & Conf. Int. (0.05) & Fairness index \\
\hline \multirow{3}{*}{802.11} & $2 \mathrm{Mbps}$ & 1240.93 & {$[1236.03 ; 1245.84]$} & 152.07 & {$[151.47 ; 152.67]$} & \\
& $11 \mathrm{Mbps}$ & 1219.97 & {$[1203.54 ; 1236.39]$} & 149.50 & {$[147.49 ; 151.51]$} & 0.7676374 \\
& Total & 2460.91 & {$[2447.07 ; 2474.74]$} & 301.58 & {$[299.88 ; 303.27]$} & \\
\hline \multirow{3}{*}{ PAS } & $2 \mathrm{Mbps}$ & 816.51 & {$[811.19 ; 821.83]$} & 100.06 & {$[99.41 ; 100.71]$} & \\
& $11 \mathrm{Mbpss}$ & 3046.88 & {$[3023.13 ; 3070.62]$} & 373.39 & {$[370.48 ; 376.30]$} & 0.9976767 \\
& Total & 3863.39 & {$[3843.14 ; 3883.64]$} & 473.45 & {$[470.97 ; 475.93]$} & \\
\hline
\end{tabular}

Table 4: Performance anomaly results (throughput and number of packets)

\begin{tabular}{|c|c|c|c|c|c|c|}
\hline & & Th. $(\mathrm{kbps})$ & Conf. Int. $(0.05)$ & Packets/s & Conf. Int. (0.05) & Fairness index \\
\hline \multirow{3}{*}{802.11} & $1 \mathrm{Mbps}$ & 740.60 & {$[737.31 ; 743.88]$} & 90.76 & {$[90.36 ; 91.16]$} & \multirow{2}{*}{} \\
& $11 \mathrm{Mbps}$ & 726.45 & {$[710.65 ; 742.24]$} & 89.03 & {$[87.09 ; 90.96]$} & 0.6497743 \\
& Total & 1467.04 & {$[1452.14 ; 1481.95]$} & 179.78 & {$[177.96 ; 181.61]$} & \\
\hline \multirow{3}{*}{ PAS } & $1 \mathrm{Mbps}$ & 461.81 & {$[457.45 ; 466.18]$} & 56.59 & {$[56.06 ; 57.13]$} & \\
& $11 \mathrm{Mbps}$ & 2941.32 & {$[2910.81 ; 2971.83]$} & 360.46 & {$[356.72 ; 364.19]$} & 0.9999946 \\
& Total & 3403.13 & {$[3375.51 ; 3430.75]$} & 417.05 & {$[413.67 ; 420.44]$} & \\
\hline
\end{tabular}

Table 5: Performance anomaly results (throughput and number of packets) 
The difference between the theoretical results (Table 10) and the simulation results can be explained by the backoff algorithm present in the IEEE 802.11 MAC. Indeed, the backoff algorithm does not provide a TDMA-like access to the medium. When there are only two stations, each station can access successively the medium. In the case of PAS, the fast station will first aggregate its packets during its transmission time and when its transmission time elapses, it will send its packets classically with IEEE 802.11 if it accesses successively to the medium. Therefore, this feature of PAS reduces the throughput of the fast station because it does not always aggregate its packets. This reduction can be worsened when the slow station sends also successive packets. The difference between the analytical results and the simulation results increases when the difference in the data rate of the two stations increases.

\begin{tabular}{|c|c|c|c|c|c|c|}
\hline & & Th. (kbps) & Conf. Int. $(0.05)$ & Packets/s & Conf. Int. (0.05) & Fairness index \\
\hline \multirow{5}{*}{802.11} & $1 \mathrm{Mbps}$ & 423.08 & {$[415.67 ; 430.49]$} & 51.85 & {$[50.94 ; 52.76]$} & \\
& $2 \mathrm{Mbps}$ & 413.68 & {$[403.86 ; 423.50]$} & 50.70 & {$[49.49 ; 51.90]$} & \\
& $5.5 \mathrm{Mbps}$ & 401.80 & {$[389.96 ; 413.65]$} & 49.24 & {$[47.79 ; 50.69]$} & 0.6598870 \\
& $11 \mathrm{Mbps}$ & 392.09 & {$[379.93 ; 404.26]$} & 48.05 & {$[46.56 ; 49.54]$} & \\
& Total & 1630.66 & {$[1614.28 ; 1647.04]$} & 199.84 & {$[197.83 ; 201.84]$} & \\
\hline \multirow{5}{*}{ PAS } & $1 \mathrm{Mbps}$ & 236.02 & {$[230.10 ; 241.94]$} & 28.92 & {$[28.20 ; 29.65]$} & \\
& $2 \mathrm{Mbps}$ & 376.81 & {$[366.19 ; 387.42]$} & 46.18 & {$[44.88 ; 47.48]$} & 0.9972993 \\
& $5.5 \mathrm{Mbps}$ & 943.25 & {$[917.63 ; 968.88]$} & 115.59 & {$[112.45 ; 118.74]$} & \\
& $11 \mathrm{Mbps}$ & 1499.68 & {$[1453.82 ; 1545.55]$} & 183.78 & {$[178.16 ; 189.41]$} & \\
& Total & 3055.77 & {$[3021.34 ; 3090.19]$} & 374.48 & {$[370.26 ; 378.70]$} & \\
\hline
\end{tabular}

Table 6: Performance anomaly results (throughput and number of packets)

\begin{tabular}{|c|c|c|c|c|c|c|}
\hline & & Th. (kbps) & Conf. Int. $(0.05)$ & Packets/s & Conf. Int. (0.05) & Fairness index \\
\hline \multirow{5}{*}{802.11} & $1 \mathrm{Mbps}$ & 260.71 & {$[251.58 ; 269.83]$} & 31.95 & {$[30.83 ; 33.07]$} & \\
& $1 \mathrm{Mbps}$ & 253.68 & {$[244.85 ; 262.52]$} & 31.09 & {$[30.01 ; 32.17]$} & \\
& $1 \mathrm{Mbps}$ & 259.36 & {$[250.78 ; 267.95]$} & 31.78 & {$[30.73 ; 32.84]$} & 0.8222611 \\
& $11 \mathrm{Mbps}$ & 267.21 & {$[256.25 ; 278.18]$} & 32.75 & {$[31.40 ; 34.09]$} & \\
& Total & 1040.97 & {$[1030.81 ; 1051.13]$} & 127.57 & {$[126.32 ; 128.81]$} & \\
\hline \multirow{5}{*}{ PAS } & $1 \mathrm{Mbps}$ & 213.50 & {$[206.55 ; 220.46]$} & 26.16 & {$[25.31 ; 27.02]$} & \\
& $1 \mathrm{Mbps}$ & 210.30 & {$[202.72 ; 217.88]$} & 25.77 & {$[24.84 ; 26.70]$} & \\
& $1 \mathrm{Mbps}$ & 202.45 & {$[193.29 ; 211.61]$} & 24.81 & {$[23.69 ; 25.93]$} & 0.9980227 \\
& $11 \mathrm{Mbps}$ & 1540.59 & {$[1488.93 ; 1592.24]$} & 188.80 & {$[182.47 ; 195.13]$} & \\
& Total & 2166.84 & {$[2120.97 ; 2212.71]$} & 265.54 & {$[259.92 ; 271.17]$} & \\
\hline
\end{tabular}

Table 7: Performance anomaly results (throughput and number of packets) 


\begin{tabular}{|c|c|c|c|c|c|c|}
\hline & & Th. (kbps) & Conf. Int. $(0.05)$ & Packets/s & Conf. Int. $(0.05)$ & Fairness index \\
\hline \multirow{5}{*}{802.11} & $1 \mathrm{Mbps}$ & 330.53 & {$[320.45 ; 340.61]$} & 40.51 & {$[39.27 ; 41.74]$} & \\
& $1 \mathrm{Mbps}$ & 345.51 & {$[336.32 ; 354.70]$} & 42.34 & {$[41.22 ; 43.47]$} & \\
& $5.5 \mathrm{Mbps}$ & 341.89 & {$[328.66 ; 355.13]$} & 41.90 & {$[40.28 ; 43.52]$} & 0.6822219 \\
& $11 \mathrm{Mbps}$ & 332.60 & {$[319.99 ; 345.20]$} & 40.76 & {$[39.21 ; 42.30]$} & \\
& Total & 1350.53 & {$[1335.64 ; 1365.43]$} & 165.51 & {$[163.68 ; 167.33]$} & \\
\hline \multirow{5}{*}{ PAS } & $1 \mathrm{Mbps}$ & 208.13 & {$[201.54 ; 214.72]$} & 25.51 & {$[24.70 ; 26.31]$} & \\
& $1 \mathrm{Mbps}$ & 214.23 & {$[208.10 ; 220.35]$} & 26.25 & {$[25.50 ; 27.00]$} & \multirow{2}{*}{0.9991965} \\
& $5.5 \mathrm{Mbps}$ & 949.87 & {$[922.42 ; 977.31]$} & 116.41 & {$[113.04 ; 119.77]$} & \\
& $11 \mathrm{Mbps}$ & 1510.32 & {$[1465.07 ; 1555.58]$} & 185.09 & {$[179.54 ; 190.63]$} & \\
& Total & 2882.55 & {$[2848.88 ; 2916.21]$} & 353.25 & {$[349.13 ; 357.38]$} & \\
\hline
\end{tabular}

Table 8: Performance anomaly results (throughput and number of packets)

Tables 6, 7 and 8 show the simulation results with four stations transmitting respectively at $\{1,2,5.5,11\} \mathrm{Mbps}$, at $\{1,1,1,11\} \mathrm{Mbps}$ and at $\{1,1,5.5,11\} \mathrm{Mbps}$. From these results, one can see that the aggregate throughput of PAS is always greater than the aggregate throughput of 802.11. The throughput and the number of packets for the fast stations (especially at 11Mbps) with PAS remain almost the same in the different tables. This is because the time accorded to each station to send its packets is based on the slowest packet time transmission. The fairness index also shows that PAS is fair in terms of medium occupancy.

\subsection{Reactivity}

A way to test the reactivity of PAS is to introduce the well-known Auto-Rate Fallback (ARF) mechanism used by wireless stations to adapt their transmission rate to the channel conditions. We have implemented the ARF mechanism to see the behavior of PAS when the transmission rates of stations vary in time. The simulation is done using two emitters with one station moving away from the other. Figure 2 shows the simulation results with PAS and 802.11. We can see from this figure that when using PAS, the throughput of the fast station remains constant, while the throughput of the moving station decreases. With IEEE 802.11, the throughput of the two emitters decreases.

\subsection{Delay}

In this section we present a simulation of 20 seconds with 2 emitters: one with a data rate of $11 \mathrm{Mbps}$ and the other with a data rate of $1 \mathrm{Mbps}$. During this simulation we compute the inter-burst time. An inter-burst time is defined as the time between the end of a burst and the beginning of another burst from the same station. For the station transmitting at the lower data rate a burst consists always in a single packet. For the station transmitting at 


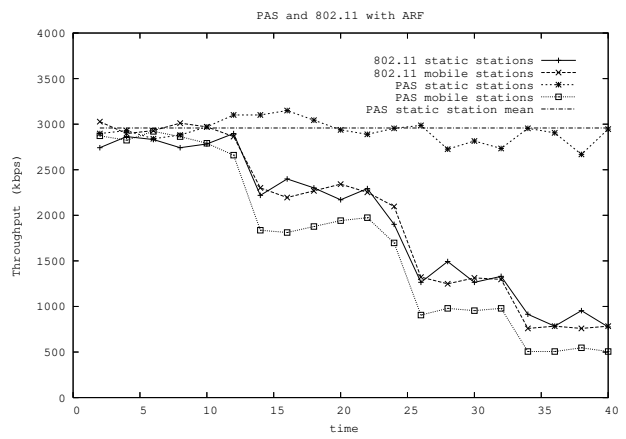

Figure 2: PAS implemented with ARF

\begin{tabular}{|l|c|c|}
\hline & Nb bursts & Avg inter-burst \\
\hline FAST & 5911 & $9867.70 \mu \mathrm{s}$ \\
\hline SLOW & 6004 & $8776.46 \mu \mathrm{s}$ \\
\hline
\end{tabular}

Table 9: PAS: delay

the higher data rate, a burst can be either a real packet burst (several aggregated packets) or a single packet if the wireless station accesses the medium immediately after a burst.

Table 9 gives the number of sent bursts and the average inter-burst time for the two stations. One can see that IEEE 802.11 provides a fair access to the medium, since the number of bursts for the slow and the fast stations is nearly the same. The table also shows that the average inter-burst time is close to the packet transmission time of the slow station $(8576 \mu s)$.

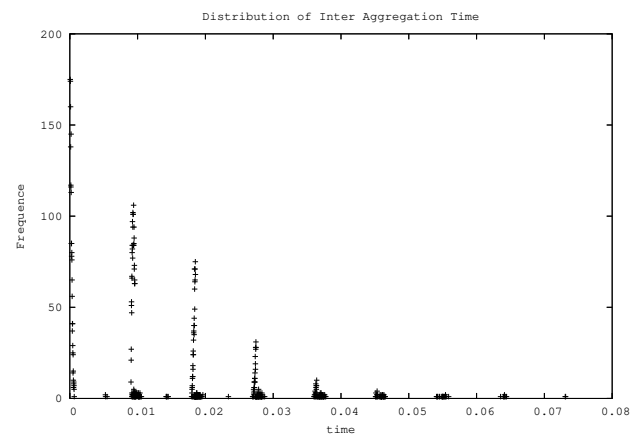

Figure 3: Inter-burst time distribution for the fast station

$\mathrm{RR} \mathrm{n}^{\circ} 0123456789$ 
Figure 3 shows the inter-burst time distribution for the fast station. One can easily see that the medium access provided by the backoff algorithm is not really a TDMA-like access due to the peak close to 0 in the figure. We can also see from this figure that the presence of successive peaks shows that the slow station can send many successive packets. This confirms what we claim in Section 5 about the difference between simulation and analytical results. In this figure the difference (in time) between two peaks is close to the packet duration of the slow station.

Figure 4 shows the inter-burst time distribution for the slow station. One can see from the figure that the average inter-burst time is close to the time needed by the fast station to transmit aggregated packets. The distribution presented in his figure is completely different from the one presented in previous figure (Figure 3). The reason is that even if the fast station can send successive packets, it is just for the transmission of a single packet and not for a burst. This also explains that the average inter-burst time of the slow station is smaller than the one of the fast station.

In both figures (Figure 3 and Figure 4), the points close to 0 means that there is a considerable number of packets that are send successively with the backoff algorithm of IEEE 802.11. Such a feature reduces the performances of PAS.

\subsection{Effect of $\alpha$}

In this section, we investigate the effect of the $\alpha$ parameter on the performance of PAS. We simulate two emitters transmitting 1000 bytes of data at $11 \mathrm{Mbps}$ and at $5.5 \mathrm{Mbps}$. The simulation is carried out with and without the use of $\alpha$. One can see from Table 10 that in this specific simulation, when $\alpha$ is not used, there is no aggregation. Indeed, in this case the condition $t \_m y \_l e f t-t \_m y \_$packet $>0$ never holds for the fast station, thus it does not perform any aggregation.

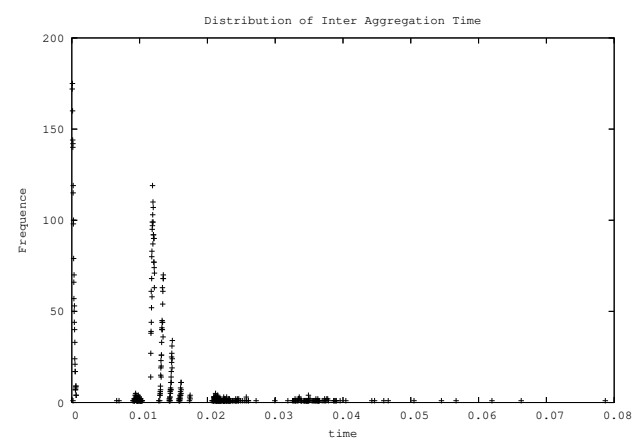

Figure 4: Inter-burt time distribution for the slow station 


\begin{tabular}{|l|c|c|c|}
\hline & & Th. (kbps) & Conf. Int. $(0.05)$ \\
\hline \multirow{3}{*}{ PAS w/o $\alpha$} & $5.5 \mathrm{Mbps}$ & 2147.31 & {$[2137.62 ; 2157.01]$} \\
& 11Mbps & 2131.51 & {$[2119.42 ; 2143.60]$} \\
& Total & 4278.83 & {$[4269.92 ; 4287.74]$} \\
\cline { 3 - 4 } & Index & \multicolumn{3}{|c|}{0.9582439} \\
\hline \multirow{3}{*}{ PAS } & $5.5 \mathrm{Mbps}$ & 1769.89 & {$[1761.23 ; 1778.54]$} \\
& $11 \mathrm{Mbps}$ & 2943.07 & {$[2927.82 ; 2958.32]$} \\
& Total & 4712.96 & {$[4703.02 ; 4722.91]$} \\
\cline { 3 - 4 } & Index & \multicolumn{3}{|c|}{0.9978824} \\
\hline
\end{tabular}

Table 10: The influence of $\alpha$ on performances

We have also simulated a scenario with four emitters, respectively at $1,2,5.5$ and 11 Mbps. From Table 10 and Table 11] we can see that $\alpha$ increases fairness and efficiency. Indeed, when $\alpha$ is used, the proportion of medium occupancy for the fast stations is increased.

\subsection{Effect of $t$ rate}

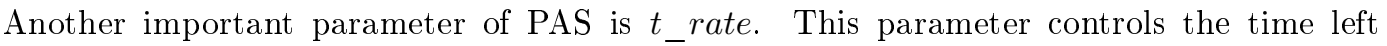
for an aggregated transmission. It increases or reduces the aggregated transmission time, depending on the ratio between payload and the header. Table 12 gives the results of simulation runs with two emitters, one transmitting at $11 \mathrm{Mbps}$ with packets of 100 bytes length, the other transmitting at $5.5 \mathrm{Mbps}$ with packets of 1000 bytes length. One can see from this table that $t$ _rate improves the global throughput of the network, but this overall throughput is smaller than in the case of IEEE 802.11. There are several possibilities to

\begin{tabular}{|l|c|c|c|}
\hline & & Th. (kbps) & Conf. Int. (0.05) \\
\hline & $1 \mathrm{Mbps}$ & 278.47 & {$[271.66 ; 285.28]$} \\
& $2 \mathrm{Mbps}$ & 283.95 & {$[274.67 ; 293.23]$} \\
PAS & $5.5 \mathrm{Mbps}$ & 880.56 & {$[857.80 ; 903.32]$} \\
w/o $\alpha$ & $11 \mathrm{Mbps}$ & 1484.19 & {$[1438.28 ; 1530.10]$} \\
& Total & 2927.17 & {$[2893.26 ; 2961.08]$} \\
\cline { 2 - 4 } & Index & \multicolumn{2}{|c|}{0.9804155} \\
\hline \multirow{3}{*}{ PAS } & 1Mbps & 236.02 & {$[230.10 ; 241.94]$} \\
& 2Mbps & 376.81 & {$[366.19 ; 387.42]$} \\
& $5.5 \mathrm{Mbps}$ & 943.25 & {$[917.63 ; 968.88]$} \\
& $11 \mathrm{Mbps}$ & 1499.68 & {$[1453.82 ; 1545.55]$} \\
& Total & 3055.77 & {$[3021.34 ; 3090.19]$} \\
\cline { 2 - 3 } & Index & \multicolumn{3}{|c|}{0.9972993} \\
\hline
\end{tabular}

Table 11: The influence of $\alpha$ on performances 


\begin{tabular}{|l|c|c|c|}
\hline & & Th.(kbps) & Conf. Int \\
\hline \multirow{3}{*}{802.11} & $11 \mathrm{Mbps}$ & 308.37 & {$[299.76 ; 316.98]$} \\
& $5.5 \mathrm{Mbps}$ & 2631.15 & {$[2586.04 ; 2676.25]$} \\
& Total & 2939.52 & {$[2898.72 ; 2980.32]$} \\
\cline { 3 - 4 } & Index & \multicolumn{2}{|c|}{0.8140598} \\
\hline \multirow{3}{*}{ PAS } & $11 \mathrm{Mbps}$ & 458.99 & {$[446.99 ; 470.98]$} \\
& $5.5 \mathrm{Mbps}$ & 2344.64 & {$[2313.36 ; 2375.92]$} \\
& Total & 2803.63 & {$[2778.27 ; 2828.98]$} \\
\cline { 3 - 4 } & Index & \multicolumn{3}{|c|}{0.9363749} \\
\hline \multirow{2}{*}{ PAS w/o } & $11 \mathrm{Mbps}$ & 816.43 & {$[801.25 ; 831.60]$} \\
\multirow{2}{*}{ t_rate } & $5.5 \mathrm{Mbps}$ & 1668.27 & {$[1629.82 ; 1706.71]$} \\
& Total & 2484.69 & {$[2456.72 ; 2512.66]$} \\
\cline { 3 - 4 } & Index & \multicolumn{3}{|c}{0.9636280} \\
\hline
\end{tabular}

Table 12: The influence of $t_{-}$rate on performances

\begin{tabular}{|l|c|c|c|}
\hline & & $t \_p \_m a x(\mu s)$ & $t \_m y \_$packet $(\mu s)$ \\
\hline \multirow{2}{*}{ PAS } & $5.5 \mathrm{Mbps}$ & $248-954$ & $320-1716$ \\
& $11 \mathrm{Mbps}$ & $248-1716$ & $285-954$ \\
\hline
\end{tabular}

Table 13: PAS with different packet sizes

improve the use of $t$ rate. For instance, if $t$ rate $\leq 1$, setting $t$ my the aggregated sending if a small packet was sent. The problem by using this scheme is that when a small packet from upper layer arrives (such as ACK from TCP protocol), it always penalizes the wireless station when it gains the access to the medium.

One can see from Table 12 that $t_{\text {_rate }}$ has a negative impact on fairness. This because the $t_{\text {_rate }}$ is used to reduce the aggregation time. In this particular scenario, it appears that there is a tradeoff between fairness and efficiency. We argue that PAS provides this good tradeoff, as Figure 5 and Figure 6 confirm. One can see from these figures that when using the $t$ rate, PAS is not as efficient as IEEE 802.11 for small values of $t$ rate, however,

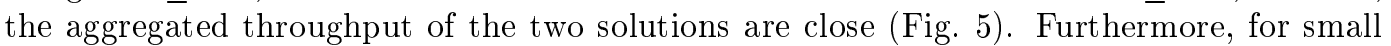
values of $t$ rate, the fairness index of PAS using $t$ rate is lower than the fairness index of PAS not using $t$ rate, however, they are very close (Fig. [6).

\subsection{Using dynamic packet sizes}

In this section we have tested our protocol with different packet sizes. Packets are generated at each node with a uniform distribution between 550 bytes and 1450 bytes. Table 13 shows the variation of $t \_p \_m a x$ and $t \_m y \_p a c k e t$ during the simulation. One can see from this table that the difference between the maximum values and the minimum values of $t \_p \_$max and $t \_m y \_p a c k e t$ may be high. 
Table 14 shows the average throughput obtained in previous simulations. One can see that PAS is efficient and fair when using a uniform distribution for the packet size. This behavior of PAS is possible because the number of packets to aggregate is not known a priori and is computed dynamically at the arrival of each new packet.

\subsection{Comparison with some other solutions}

We have also compared PAS, our proposal, to other solution. The results we obtained are presented hereafter.

\subsubsection{A simple backoff-based approach}

We have developed a simple backoff-based approach to solve the performance anomaly. This approach is based on the solution proposed by Heusse et $\mathrm{Al}$. [5]. The size of the contention window $(\mathrm{CW})$ is adapted in the following way: $C W=C W * \frac{11 e 6}{\text { dataRate }}$. In the simulations,

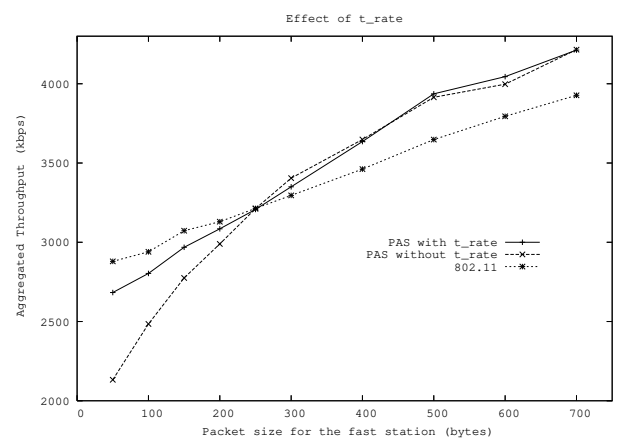

Figure 5: Aggregated throughput depending on the packet size

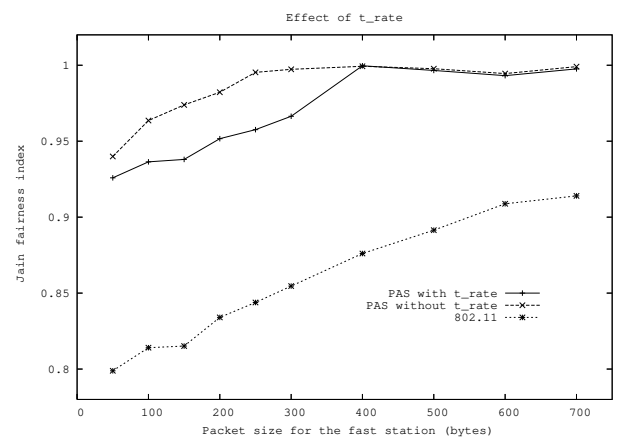

Figure 6: Fairness index depending on the packet size

$\mathrm{RR} \mathrm{n}^{\circ} 0123456789$ 


\begin{tabular}{|l|c|c|c|}
\hline & & Th. (kbps) & Conf. Int. (0.05) \\
\hline \multirow{3}{*}{802.11} & $5.5 \mathrm{Mbps}$ & 2075.67 & {$[2065.93 ; 2085.41]$} \\
& $11 \mathrm{Mbps}$ & 2073.35 & {$[2059.62 ; 2087.08]$} \\
& Total & 4149.03 & {$[4139.91 ; 4158.15]$} \\
\cline { 3 - 4 } & Index & \multicolumn{3}{|c|}{0.9593866} \\
\hline \multirow{3}{*}{ PAS } & $5.5 \mathrm{Mbps}$ & 1741.43 & {$[1733.81 ; 1749.05]$} \\
& $11 \mathrm{Mbps}$ & 2782.73 & {$[2769.18 ; 2796.27]$} \\
& Total & 4524.16 & {$[4514.01 ; 4534.31]$} \\
\cline { 3 - 4 } & Index & \multicolumn{3}{|c|}{0.9993147} \\
\hline
\end{tabular}

Table 14: PAS with packet sizes uniformly distributed

the size of packets is uniformly distributed in the interval $[550 ; 1450]$ bytes and there are two emitters, one at transmitting at $5.5 \mathrm{Mbps}$ and the other at $11 \mathrm{Mbps}$. Table 15 gives the average throughput as the average fairness index. One can see that this approach is efficient, but not as efficient as our solution (see results for PAS in Table 14). This is due to the overhead introduced for each packet by the backoff algorithm. Another problem of this approach is when small packets are sent by the fast station. In this case, the performance of the backoff-based approach decreases.

\subsubsection{Packet Division approach}

We have also tested the packet division approach proposed by Iannone et Al. 6]. The simulations are carried out with two emitters, one transmitting at $11 \mathrm{Mbps}$ and the other at $5.5 \mathrm{Mbps}$. The packet size of the fast station is set to 1500 bytes, while the packet size of the slow station is but set to 727 bytes due to the fragmentation required in this solution. In the simulation, the two packet sizes are set to 1500 bytes with PAS. Table 16 shows the results of these simulations. One can see from this table that the packet division approach is less efficient, due to the overhead introduced by the backoff and the header. It would also be trivial to show that when all wireless stations in the network use a small data rate, the network performance is reduced because the packet fragmentation increases the payload/header ratio.

\begin{tabular}{|l|c|c|c|}
\hline & & Th. (kbps) & Conf. Int. (0.05) \\
\hline \multirow{3}{*}{ Backoff } & $5.5 \mathrm{Mbps}$ & 1327.62 & {$[1314.12 ; 1341.11]$} \\
adaptation & $11 \mathrm{Mbps}$ & 3061.40 & {$[3045.48 ; 3077.32]$} \\
& Total & 4389.02 & {$[4381.08 ; 4396.96]$} \\
\cline { 3 - 4 } & Index & \multicolumn{3}{|c|}{0.9590798} \\
\hline
\end{tabular}

Table 15: Backoff-based approach 


\begin{tabular}{|l|c|c|c|}
\hline & & Th. $(\mathrm{kbps})$ & Conf. Int. $(0.05)$ \\
\hline Packet & $5.5 \mathrm{Mbps}$ & 1779.97 & {$[1771.88 ; 1788.06]$} \\
division & $11 \mathrm{Mbps}$ & 2377.61 & {$[2365.28 ; 2389.94]$} \\
& Total & 4157.59 & {$[4149.42 ; 4165.75]$} \\
\cline { 3 - 4 } & Index & \multicolumn{3}{|c|}{0.9960047} \\
\hline \multirow{3}{*}{ PAS } & $5.5 \mathrm{Mbps}$ & 1772.22 & {$[1764.16 ; 1780.29]$} \\
& $11 \mathrm{Mbps}$ & 2936.01 & {$[2922.13 ; 2949.89]$} \\
& Total & 4708.24 & {$[4698.97 ; 4717.51]$} \\
\cline { 3 - 4 } & Index & \multicolumn{3}{|c|}{0.9980492} \\
\hline
\end{tabular}

Table 16: Packet division approach

\begin{tabular}{|c|c|c|c|}
\hline & & Th. (kbps) & Conf. Int. $(0.05)$ \\
\hline \multirow{4}{*}{ FIXED } & $5.5 \mathrm{Mbps}$ & 1972.00 & {$[1955.38 ; 1988.62]$} \\
& $11 \mathrm{Mbps}$ & 2988.83 & {$[2959.72 ; 3017.94]$} \\
& Total & 4960.84 & {$[4947.75 ; 4973.92]$} \\
\cline { 3 - 4 } & Index & \multicolumn{2}{|c|}{0.9999999} \\
\hline
\end{tabular}

Table 17: Fixed aggregation time

\subsubsection{Fixed time aggregation approach}

To carry out this simulation we have modified our implementation of PAS, introducing a fixed $t \_p \_\max =8000 \mu \mathrm{s}$. With this value, a node transmitting a $1500 \mathrm{bytes}$ data at $1 \mathrm{Mbps}$ can send only one packet. One can see from Table 17 comparing to Table 14 that the aggregation using fixed time is more efficient than our approach. This is due to the fact that, differently from PAS, the aggregation is always used. On the other hand, this permanent aggregation implies longer delays between bursts. Table 18 shows the number of bursts and the average time between two bursts emitted by the same station. One can see from this table that the delay induced by PAS is much smaller compared to the other approach.

\begin{tabular}{|l|c|c|c|}
\hline & & Nb burts & Avg inter-access \\
\hline & $5.5 \mathrm{Mbps}$ & 7123 & $11230.07 \mu \mathrm{s}$ \\
FIXED & $11 \mathrm{Mbps}$ & 6666 & $12000.80 \mu \mathrm{s}$ \\
\hline & $5.5 \mathrm{Mbps}$ & 19570 & $4087.80 \mu \mathrm{s}$ \\
PAS & $11 \mathrm{Mbps}$ & 19346 & $4135.11 \mu \mathrm{s}$ \\
\hline
\end{tabular}

Table 18: Performance anomaly delay results 


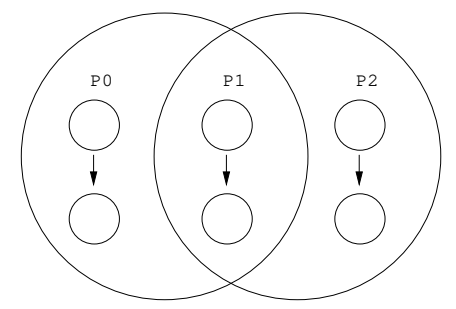

Figure 7: The 3 pairs scenario

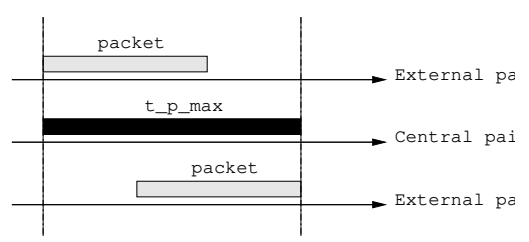

Figure 8: The medium occupancy perceived by the central pair

\subsection{PAS in a multi-hop context}

\subsubsection{3 pairs scenario}

Since all the mechanisms in PAS are fully distributed, PAS can also work in a multi-hop context, where the wireless stations do not perceive the same medium occupancy. If we consider the scenario depicted in Figure 7 we can see that the external pairs are fully independent. In this scenario, the central pair accesses the medium 95\% less than the external pairs, as demonstrated by Chaudet et Al. [1]. The medium occupancy perceived by the central pair is given in Figure [8. One can see from this figure that the value of $t \_p \_$max for the central pair can be at most equal to $t \_p 1+t \_p 2$, where $t \_p i_{i \in\{1,2\}}$ is the time needed for the pair $i$ to transmit its packet. It is important to remark that here the maximum medium occupancy time does not specifically correspond to a packet transmission time. Table 19 shows the results on the 3 pairs scenario where the external pairs send 1000 bytes of data at $2 \mathrm{Mbps}$ and the central pair sends 1000 bytes of data at $11 \mathrm{Mbps}$.

One can see from this table that even if PAS does not solve the problem, the throughput of the central pair is highly improved. Nevertheless, in this scenario a temporal fairness can not solve the problem and it seems necessary to modify the 802.11 medium access control in order to provide each node the same probability to access the medium.

\subsubsection{Hidden terminals}

In Section 4 we have proposed a RTS/CTS mechanism for PAS. Table 20 evaluates this mechanism. In this simulation we simulate two hidden nodes. The RTS/CTS threshold 
is set to 200 bytes and packet size to 1000 bytes. One can see from this table that the RTS/CTS mechanism of PAS is close to the original 802.11's one.

In order to evaluate the performance of PAS in a multi-hop context with aggregation, one of the hidden nodes uses a data rate of $x$, where $x \in\{1,2,5.5\} \mathrm{Mbps}$, while the other sends at $11 \mathrm{Mbps}$. Tables 21, 22 and 23 show the simulation results from these simulations. We can see from these tables that PAS is more efficient and fairer than 802.11 when one of the pairs has a data rate of 1 or $2 \mathrm{Mbps}$. This is because more aggregated packet can be sent by the fast station. On the other, we see that the results of PAS at 11 and $5.5 \mathrm{Mbps}$ are very close to the ones of 802.11 (Table 21). Since the time duration in the RTS corresponds to the transmission time of the packet to send, then a collision is likely to occur on the second packet of the aggregated series. With 11 and $5.5 \mathrm{Mbps}, t_{-} m y \_l e f t$ is not large enough to aggregate the packet again, whereas with 11 and $2 \mathrm{Mbps}$ (Table 22) or 11 and $1 \mathrm{Mbps}$ (Table 23), $t$ _my_left is large enough to aggregate the packet that has collided. In these two latter configurations, after some collisions, the contention window of the slow station is large enough to allow the aggregated sending of the fast station.

Table 24] shows the simulation results for two hidden nodes transmitting at 1 and $11 \mathrm{Mbps}$, with a packet size uniformly distributed between $[550 ; 1450]$ bytes. In this simulation we set the RTS threshold to 1000 bytes. One can see from these results that, even with different packet sizes, thus with a different RTS/CTS policy for each packet (the RTS/CTS is not always activated), PAS is more efficient and fair than 802.11. Note that in this simulation,

\begin{tabular}{|l|l|c|c|}
\hline & & Th. $(\mathrm{kbps})$ & Conf. Int. \\
\hline \multirow{3}{*}{ PAS } & P0 & 1592.49 & {$[1584.16 ; 1600.82]$} \\
& P1 & 102.21 & {$[68.28 ; 136.15]$} \\
& P2 & 1592.49 & {$[1584.09 ; 1600.89]$} \\
\hline \multirow{3}{*}{802.11} & P0 & 1634.15 & {$[1632.03 ; 1636.27]$} \\
& P1 & 6.44 & {$[1.78 ; 11.11]$} \\
& P2 & 1632.86 & {$[1630.23 ; 1635.49]$} \\
\hline
\end{tabular}

Table 19: Results on 3 pairs scenario

\begin{tabular}{|l|c|c|c|}
\hline & & Th. $(\mathrm{kbps})$ & Conf. Int. $(0.05)$ \\
\hline \multirow{3}{*}{802.11} & $11 \mathrm{Mbps}$ & 1821.80 & {$[1770.05 ; 1873.55]$} \\
RTS/CTS & $11 \mathrm{Mbps}$ & 1756.10 & {$[1704.39 ; 1807.82]$} \\
& Total & 3577.91 & {$[3572.61 ; 3583.20]$} \\
\cline { 3 - 4 } & Index & \multicolumn{3}{|c|}{0.9996629} \\
\hline \multirow{3}{*}{ PAS } & $11 \mathrm{Mbps}$ & 1760.83 & {$[1704.99 ; 1816.67]$} \\
RTS/CTS & $11 \mathrm{Mbps}$ & 1818.07 & {$[1761.90 ; 1874.23]$} \\
& Total & 3578.90 & {$[3573.59 ; 3584.21]$} \\
\cline { 3 - 4 } & Index & \multicolumn{3}{|c|}{0.9997443} \\
\hline
\end{tabular}

Table 20: RTS/CTS validation 


\begin{tabular}{|l|c|c|c|}
\hline & & Th. (kbps) & Conf. Int. $(0.05)$ \\
\hline & $5.5 \mathrm{Mbps}$ & 1558.51 & {$[1518.96 ; 1598.06]$} \\
802.11 & $11 \mathrm{Mbps}$ & 1503.17 & {$[1450.54 ; 1555.80]$} \\
RTS/CTS & Total & 3061.68 & {$[3048.00 ; 3075.36]$} \\
\cline { 3 - 4 } & Index & \multicolumn{3}{|c|}{0.9795797} \\
\hline \multirow{3}{*}{ PAS } & $5.5 \mathrm{Mbps}$ & 1584.43 & {$[1539.41 ; 1629.44]$} \\
RTS/CTS & $11 \mathrm{Mbps}$ & 1463.86 & {$[1404.63 ; 1523.08]$} \\
& Total & 3048.28 & {$[3033.50 ; 3063.06]$} \\
\cline { 3 - 4 } & Index & \multicolumn{3}{|c|}{0.9733833} \\
\hline
\end{tabular}

Table 21: RTS/CTS with 5.5 and 11Mbps nodes

\begin{tabular}{|l|c|c|c|}
\hline & & Th. (kbps) & Conf. Int. $(0.05)$ \\
\hline \multirow{3}{*}{802.11} & $2 \mathrm{Mbps}$ & 1003.95 & {$[979.74 ; 1028.16]$} \\
RTS/CTS & $11 \mathrm{Mbps}$ & 1064.32 & {$[1003.33 ; 1125.30]$} \\
& Total & 2068.27 & {$[2031.39 ; 2105.14]$} \\
\cline { 3 - 4 } & Index & \multicolumn{2}{|c|}{0.8721524} \\
\hline \multirow{3}{*}{ PAS } & $2 \mathrm{Mbps}$ & 827.97 & {$[802.97 ; 852.97]$} \\
RTS/CTS & $11 \mathrm{Mbps}$ & 1526.34 & {$[1463.53 ; 1589.15]$} \\
& Total & 2354.31 & {$[2316.41 ; 2392.20]$} \\
\cline { 3 - 4 } & Index & \multicolumn{3}{|c}{0.9856836} \\
\hline
\end{tabular}

Table 22: RTS/CTS with 2 and 11Mbps nodes

\begin{tabular}{|l|c|c|c|}
\hline & & Th. (kbps) & Conf. Int. (0.05) \\
\hline \multirow{3}{*}{802.11} & 1Mbps & 670.43 & {$[658.39 ; 682.48]$} \\
RTS/CTS & T1Mbps & 663.54 & {$[611.37 ; 715.71]$} \\
& Total & 1333.98 & {$[1293.81 ; 1374.15]$} \\
\cline { 3 - 4 } & Index & \multicolumn{2}{|c|}{0.7205043} \\
\hline \multirow{3}{*}{ PAS } & $1 \mathrm{Mbps}$ & 552.45 & {$[535.59 ; 569.31]$} \\
RTS/CTS & 11Mbps & 1237.35 & {$[1161.59 ; 1313.12]$} \\
& Total & 1789.80 & {$[1730.87 ; 1848.73]$} \\
\cline { 3 - 4 } & Index & \multicolumn{3}{|c|}{0.9071351} \\
\hline
\end{tabular}

Table 23: RTS/CTS with 1 and 11Mbps nodes 


\begin{tabular}{|l|c|c|c|}
\hline & & Th. (kbps) & Conf. Int. (0.05) \\
\hline \multirow{3}{*}{802.11} & 1Mbps & 305.92 & {$[297.37 ; 314.46]$} \\
RTS/CTS & 11Mbps & 919.55 & {$[888.74 ; 950.36]$} \\
& Total & 1225.47 & {$[1200.47 ; 1250.47]$} \\
\cline { 3 - 4 } & Index & \multicolumn{2}{|c|}{0.7945568} \\
\hline \multirow{3}{*}{ PAS } & $1 \mathrm{Mbps}$ & 226.39 & {$[218.78 ; 234.00]$} \\
RTS/CTS & 11Mbps & 1304.61 & {$[1269.65 ; 1339.57]$} \\
& Total & 1531.01 & {$[1500.21 ; 1561.80]$} \\
\cline { 3 - 4 } & Index & \multicolumn{3}{|c|}{0.9493314} \\
\hline
\end{tabular}

Table 24: RTS/CTS with 1 and 11Mbps nodes with uniformly distributed packet and 1000 bytes threshold

the value of $t \_p \_$max when RTS/CTS are not used corresponds to the transmission time of the acknowledgment.

\section{Conclusion}

In this paper we propose PAS, a dynamic packet aggregation mechanism to solve the performance anomaly of 802.11. Our solution is based on the fact that the same transmission time is given to each station. This transmission time is computed dynamically and is equal to the maximum occupation time perceived on the medium. When a node has the opportunity to use the channel, it sends as many packets as the transmission time allows. The aggregation is done by waiting only for a SIFS period between the reception of an ACK and the beginning of the next transmission. To increase the dynamicity and to reduce the convergence time, the transmission time is set to 0 after each successful transmission (or burst of aggregate transmission).

We have shown, through both analytical analysis and simulation, that our protocol solves the performance anomaly in many scenarios. The aggregate throughput can be increased and the time-based fairness is almost reached in almost every of the tested configurations. We have also shown that our approach does not need extra information than that already furnished by IEEE 802.11 standard, thus it can be easily implemented. An important characteristic of our proposal is the fact that it can be also used in multi-hop networks, improving also in this scenario the performances.

\section{References}

[1] C. Chaudet, I. Guérin Lassous, E. Thierry, and B. Gaujal. Study of the impact of asymmetry and carrier sense mechanism in IEEE 802.11 multi-hops networks through a basic case. In Proceedings of the 1st ACM Workshop on Performance Evaluation 
of Wireless Ad Hoc, Sensor, and Ubiquitous Networks (PE-WASUN'04), Venice, Italy, October 2004.

[2] J. Dunn, M. Neufeld, A. Sheth, D. Grunwald, and J. Bennett. A practical cross-layer mechanism for fairness in 802.11 networks. In BROADNETS, pages 355-364, 2004.

[3] IEEE Standard for Information Technology Telecommunications and Information Exchange between Systems. Local and Metropolitan Area Network - Specific Requirements - Part 11: Wireless LAN Medium Access Control (MAC) and Physical Layer (PHY) Specifications, 1997.

[4] M. Heusse, F. Rousseau, G. Berger-Sabbatel, and A. Duda. Performance anomaly of 802.11b. In INFOCOM, 2003.

[5] M. Heusse, F. Rousseau, R. Guillier, and A. Duda. Idle sense: an optimal access method for high throughput and fairness in rate diverse wireless lans. In SIGCOMM '05, pages 121-132, New York, NY, USA, 2005. ACM Press.

[6] L. Iannone and S. Fdida. Sdt.11b : Un schéma à division de temps pour éviter l'anomalie de la couche mac $802.11 \mathrm{~b}$. In CFIP, Colloque Francophone sur l'Ingénierie des Protocoles, April.

[7] Information Sciences Institute. NS-2 network simulator. Software Package, 2003. http://www.isi.edu/nsnam/ns/

[8] Institute of Electrical and Electronics Engineers (IEEE). Ieee 802.11e/d12.0. draft supplement to part 11: Wireless medium access control (mac) and physical layer (phy) specifications: Medium access control (mac) enhancements for quality of service (qos). IEEE 802.11 WG, 2004.

[9] R. Jain. Throughput fairness index: An explanation. ATM Forum Contribution 99$0045,1999$.

[10] B Sadeghi, V. Kanodia, A. Sabharwal, and E. Knightly. Opportunistic media access for multirate ad hoc networks. In MobiCom '02, pages 24-35, New York, NY, USA, 2002. ACM Press.

[11] G. Tan and J. V. Guttag. Time-based fairness improves performance in multi-rate wlans. In USENIX Annual Technical Conference, General Track, pages 269-282, 2004. 


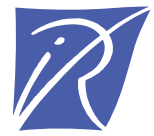

Unité de recherche INRIA Rhône-Alpes

655, avenue de l'Europe - 38334 Montbonnot Saint-Ismier (France)

Unité de recherche INRIA Futurs : Parc Club Orsay Université - ZAC des Vignes 4, rue Jacques Monod - 91893 ORSAY Cedex (France)

Unité de recherche INRIA Lorraine : LORIA, Technopôle de Nancy-Brabois - Campus scientifique

615, rue du Jardin Botanique - BP 101 - 54602 Villers-lès-Nancy Cedex (France)

Unité de recherche INRIA Rennes : IRISA, Campus universitaire de Beaulieu - 35042 Rennes Cedex (France)

Unité de recherche INRIA Rocquencourt : Domaine de Voluceau - Rocquencourt - BP 105 - 78153 Le Chesnay Cedex (France)

Unité de recherche INRIA Sophia Antipolis : 2004, route des Lucioles - BP 93 - 06902 Sophia Antipolis Cedex (France)

INRIA - Domaine de Voluceau - Rocquencourt, BP 105 - 78153 Le Chesnay Cedex (France)

http://www.inria.fr

ISSN 0249-6399 


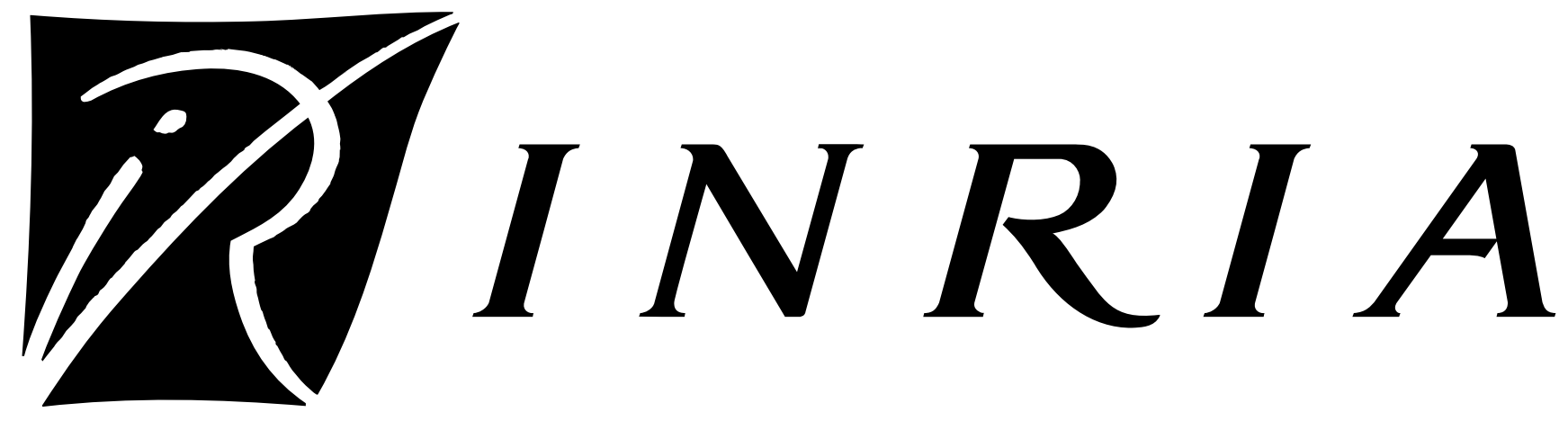


